\title{
Pragmatic randomized trial evaluating pre- operative aqueous antiseptic skin solution in open fractures (Aqueous-PREP): the feasibility of a cluster randomized crossover study
}

Sheila Sprague ${ }^{1,2^{*}}$ (D), Paige Guyatt ${ }^{1}$, Sofia Bzovsky ${ }^{1}$, Uyen Nguyen ${ }^{1}$, Mohit Bhandari ${ }^{1,2}$, Lehana Thabane ${ }^{2}$, Brad Petrisor', Herman S. Johal', Jordan Leonard', Shannon Dodds' ${ }^{1}$, Franca Mossuto', Robert V. O'Toole ${ }^{3}$, Andrea Howe ${ }^{3}$, Haley K. Demyanovich ${ }^{3}$, Megan Camara ${ }^{3}$, Nathan N. O'Hara ${ }^{3}$, Gerard P. Slobogean ${ }^{3}$ The PREP-IT Investigators

\begin{abstract}
Background: Preoperative antiseptic skin solutions are used prior to most surgical procedures; however, there is no definitive research comparing infection-related outcomes following use of the various solutions available to orthopedic trauma surgeons. The objective of this pilot study was to test the feasibility of a cluster randomized crossover trial that assesses the comparative effectiveness of a 10\% povidone-iodine solution versus a $4 \%$ chlorhexidine gluconate solution for the management of open fractures.

Methods: Two orthopedic trauma centers participated in this pilot study. Each of these clinical sites was randomized to a starting solution (povidone-iodine solution or chlorhexidine gluconate) then subsequently crossed over to the other treatment after 2 months. During the 4-month enrollment phase, we assessed compliance, enrollment rates, participant follow-up, and accurate documentation of the primary clinical outcome. Feasibility outcomes included (1) the implementation of the interventions during a run-in period; (2) enrollment of participants during two 2-month enrollment phases; (3) application of the trial interventions as per the cluster randomization crossover scheme; (4) participant follow-up; and (5) accurate documentation of the primary outcome (surgical site infection). Feasibility outcomes were summarized using descriptive statistics reported as means (standard deviation) or medians (first quartile, third quartile) for continuous variables depending on their distribution and counts (percentage) for categorical variables. Corresponding 95\% confidence intervals (Cls) were also reported.

(Continued on next page)
\end{abstract}

\footnotetext{
*Correspondence: sprags@mcmaster.ca

'Division of Orthopedic Surgery, Department of Surgery, McMaster University, Hamilton, Ontario, Canada

${ }^{2}$ Department of Health Research Methods, Evidence, and Impact, McMaster

University, Hamilton, Ontario, Canada

Full list of author information is available at the end of the article
}

(c) The Author(s). 2021 Open Access This article is licensed under a Creative Commons Attribution 4.0 International License, which permits use, sharing, adaptation, distribution and reproduction in any medium or format, as long as you give appropriate credit to the original author(s) and the source, provide a link to the Creative Commons licence, and indicate if changes were made. The images or other third party material in this article are included in the article's Creative Commons licence, unless indicated otherwise in a credit line to the material. If material is not included in the article's Creative Commons licence and your intended use is not permitted by statutory regulation or exceeds the permitted use, you will need to obtain permission directly from the copyright holder. To view a copy of this licence, visit http://creativecommons.org/licenses/by/4.0/ The Creative Commons Public Domain Dedication waiver (http://creativecommons.org/publicdomain/zero/1.0/) applies to the data made available in this article, unless otherwise stated in a credit line to the data. 
(Continued from previous page)

Results: All five of the criteria for feasibility were met. During the run-in phase, all 18 of the eligible patients identified at the two clinical sites received the correct cluster-assigned treatment. A total of 135 patients were enrolled across both sites during the 4-month recruitment phase, which equates to 92\% (95\% Cl 85.9 to 96.4\%) of eligible patients being enrolled. Compliance with the assigned treatment in the pilot study was 98\% (95\% Cl 93.5 to 99.8\%). Ninetyeight percent (95\% Cl 93.5 to 99.8\%) of participants completed the 90-day post-surgery follow-up and the primary outcome (SSI) was accurately documented for 100\% (95\% Cl 96.6 to 100.0\%) of the participants.

Conclusions: These results confirm the feasibility of a definitive study comparing antiseptic solutions using a cluster randomized crossover trial design. Building upon the infrastructure established during the pilot phase, a definitive study has been successfully initiated.

Trial registration: ClincialTrials.gov, number NCT03385304. Registered December 28, 2017.

Keywords: Pilot study, Feasibility, Open fractures, Aqueous antiseptic skin solution, Surgical site infection, Cluster crossover

\section{Key messages}

- Given that there is limited research addressing the comparative outcomes associated with various types of antiseptics in open fracture surgery and that Aqueous-PREP follows a cluster randomized crossover design, which is novel in the field of orthopedic trauma surgery, a pilot study was conducted.

- All feasibility criteria were met in the pilot study:

○ Enrollment of $92 \%$ of open fracture patients during the enrollment periods

○ $98 \%$ of participants receiving the treatment as per the cluster randomization on their initial surgery ○ Participant follow-up of $98 \%$ at 90 -day postfracture surgery

$\circ$ Accurate documentation of $100 \%$ of the primary outcome (SSI)

- Building upon the infrastructure, developed, and momentum and insights gained through the pilot study, we were able to successfully transition directly into the definitive trial phase of the Aqueous-PREP trial.

\section{Introduction}

Sterile technique and pre-operative skin cleaning with antiseptic solutions are vital components of peri-operative care for open fracture patients as they decrease surgical site infections (SSI) [1-4]. Antiseptic solutions, when applied to the skin immediately prior to surgery, are able to destroy bacteria and also diminish the amount of native skin flora and therefore are thought to reduce surgical site infection [1-4]. Skin preparation solutions are either alcohol or aqueous-based, and commonly include either an iodophor or chlorhexidine-based active ingredient.

Despite the recognized importance of applying skin preparation solutions immediately prior to surgery, there is limited research addressing the comparative outcomes associated with various types of antiseptics in open fracture surgery. Much of the existing evidence comes from research conducted in other surgical contexts. The limitations of generalizing results from other surgical settings to open fracture wound management is widely recognized, as the risk of SSI is substantially greater in fracture patients [4]. Fracture patients experience a higher potential for contamination for several reasons, including soft tissue damage following an injury, diminished blood flow to the injured limb if a tourniquet is used, the limited opportunity for additional prophylactic skin care due to the emergent nature of fracture surgery, and the risk related to inserting a metal implant that may promote bacterial biofilm growth [5].

The limited research that has investigated surgical skin preparation effectiveness in open fracture management found that povidone-iodine (an aqueous iodophor solution) could potentially provide increased protection in comparison to chlorhexidine; however, the results suggest that iodophor may reduce the odds of infection by $31 \%$ or, conversely, increase it up to $12 \%$ [6]. Thus, while there are certain chemical properties of povidone-iodine that could potentially allow it to block infection more effectively than CHG [7], it is unknown whether it should be the solution of choice for orthopedic surgeons tending to open fractures.

Given these uncertainties, the PREP-IT Investigators developed the protocol for the Aqueous-PREP (Pragmatic Randomized Trial Evaluating Pre-Operative Aqueous and Antiseptic Skin Solution in Open Fractures) trial, which aims to compare the effectiveness of an aqueous preoperative antiseptic preparation with $10 \%$ povidone-iodine (an iodophor solution) versus 4\% CHG for the management of open fractures. A ranked order for assessing effectiveness will be used, with SSI as the primary comparison and unplanned fracture-related reoperations as the secondary comparison. Aqueous-PREP follows a cluster randomized crossover design, which is novel in the field of orthopedic trauma surgery [5]. We selected cluster randomized crossover design over the parallel randomized 
controlled trial design to facilitate participant enrollment and to reduce the risk of treatment contamination $[8,9]$. Due to the novel nature of the design, we conducted a pilot study prior to initiating the Aqueous-PREP trial, with a primary objective to prove the feasibility of comparing these two solutions using cluster randomized crossover trial.

\section{Methods}

Two orthopedic trauma centers participated in this pilot study, one in Canada and one in the USA. Each of these clinical sites was randomized to a starting surgical preparation solution (povidone-iodine solution or chlorhexidine gluconate). After a run-in period and a 2-month enrollment period, they crossed over to the other treatment for an additional 2 months of enrollment. Participants were followed for 90-day post-fracture fixation surgery. Feasibility outcomes were assessed over the duration of the pilot study. The master protocol followed during the implementation of the Aqueous-PREP trial has been published [5], and the objectives and methods are described in detail within this manuscript. The trial was registered with ClincialTrials.gov, number NCT03385304. It was approved by the Hamilton Integrated Research Ethics Board (\#4336) and the Advarra Institutional Review Board (formerly Chesapeake Institutional Review Board) (\#Pro00023709).

\section{Randomization and the run-in phase}

The order of treatment allocation for each clinical site was randomly assigned by personnel at the Center for Evidence-Based Orthopedics (CEO) Methods Center using a computer-generated randomization table. Prior to initiating patient recruitment, each clinical site completed a 1-month run-in phase. During this time, the sites began using their assigned pre-operative antiseptic skin solution for eligible open fracture surgeries. This run-in period was implemented to ensure that acceptable compliance was achieved before initiating participant enrollment. Compliance was monitored by Methods Center by reviewing data submitted by study personnel at each clinical site.

\section{Enrollment phases}

Following the initial run-in phase, participant recruitment began. Each clinical site continued to use their previously assigned surgical antiseptic skin solution for all eligible open fracture surgeries over an approximate 2-month enrollment period. Participants received the allocated treatment solution for their initial fracture management surgery, as well as for repeat planned surgeries, even if the repeat surgery fell within the subsequent enrollment period that used the non-allocated solution. After the first enrollment period was complete, the sites crossed over to the opposite study solution and moved directly into the second 2-month enrollment phase. No run-in phase was implemented at the time of crossover to the second solution.

\section{Eligibility criteria}

Eligible patients were adult men and women ages 18 or older with an open fracture of the appendicular skeleton, who had received or would receive definitive fracture treatment with surgical implant(s). Patients' open fracture wound management was required to include formal surgical debridement within $72 \mathrm{~h}$ of their injury, with surgeries being performed by a participating surgeon or delegate. All patients or their proxy were required to provide informed consent, and patients were required to be enrolled within 3 weeks of their fracture.

\section{Clinical outcomes and participant follow-up}

The primary outcome was SSI at 90-day post-fracture surgery. Participants were assessed during their hospital stay, and at 6 weeks and 90-day post-fracture surgery.

\section{Pilot study outcomes and success criteria}

The primary feasibility outcome was a composite outcome of five equally weighted factors. To consider the trial "feasible," all five factors had to be determined to be feasible. These five factors were as follows:

(1) The implementation of the trial intervention during the run-in period (defined as $>90 \%$ of eligible patients receiving the allocated antiseptic solution during the run-in phase)

(2) Enrollment of participants across the two planned 2-month enrollment phases at two centers (defined as enrollment of $>75 \%$ of open fracture patients over each of the two 2-month enrollment periods)

(3) Application of the trial interventions as per the cluster randomization crossover scheme (defined as $>95 \%$ of participants receiving the treatment as per the cluster randomization on their initial surgery)

(4) Achievement of high rates of participant follow-up (defined as participant follow-up of $>95 \%$ at 90 -day post-fracture surgery)

(5) Accurate documentation of the primary outcome (surgical site infection (SSI) defined as less than 5\% error rate in the documentation of the primary outcome).

A definitive Aqueous-PREP cluster randomized crossover trial would be considered feasible if the clinical sites participating in the pilot phase collectively reached all five of these thresholds.

\section{Statistical analysis Sample size}

Since the feasibility objectives in our pilot study did not lend themselves to traditional quantitative sample size calculations, we selected a sample size of 2 clinical sites 
and an enrollment period of 4 months. This enrollment period allowed each clinical site to enroll patients into each of the two treatment groups, and we estimated that between 60 and 100 participants would be enrolled across the two sites. This approach allowed us to assess the feasibility of successfully implementing a large definitive trial.

\section{Statistical analysis of outcomes}

We followed the CONSORT extensions to pilot and cluster trials when reporting the results of this pilot study $[6,8]$. Participant demographic characteristics, fracture characteristics, and feasibility outcomes were summarized using descriptive statistics reported as means (standard deviation (SD)) or medians (first quartile, third quartile) for continuous variables depending on their distribution and counts (percentage) for categorical variables. Corresponding 95\% confidence intervals (CIs) were also reported. All data analyses were conducted using R (version 4.0.0, R Foundation for Statistical Computing, Vienna, Austria).

\section{Results}

Feasibility criteria 1: Run-in phase

Two orthopedic trauma centers participated in the Aqueous-PREP pilot trial: Hamilton Health Sciences General Site (HGH) in Hamilton, Ontario, Canada, and the R Adams Cowley Shock Trauma Center (STC) in Baltimore, MD, USA. These pilot sites were randomized to one of the two skin preparation solutions and completed successful run-in phases. During the run-in phase, all eligible patients at both sites were treated with the appropriate surgical skin preparation solutions; STC prepped 15 out of 15 patients correctly and HGH prepped 3 out of 3 patients correctly for a total of 18 patients. After completing a successful run-in period, the sites began patient recruitment (Fig. 1).

\section{Feasibility criteria 2: Participant enrollment}

Over the course of the two 2-month enrollment phases (April 3, 2018 to August 21, 2018), STC screened 167 patients, 76 of which were ineligible and excluded. Ninety-one screened patients were eligible, of which 83

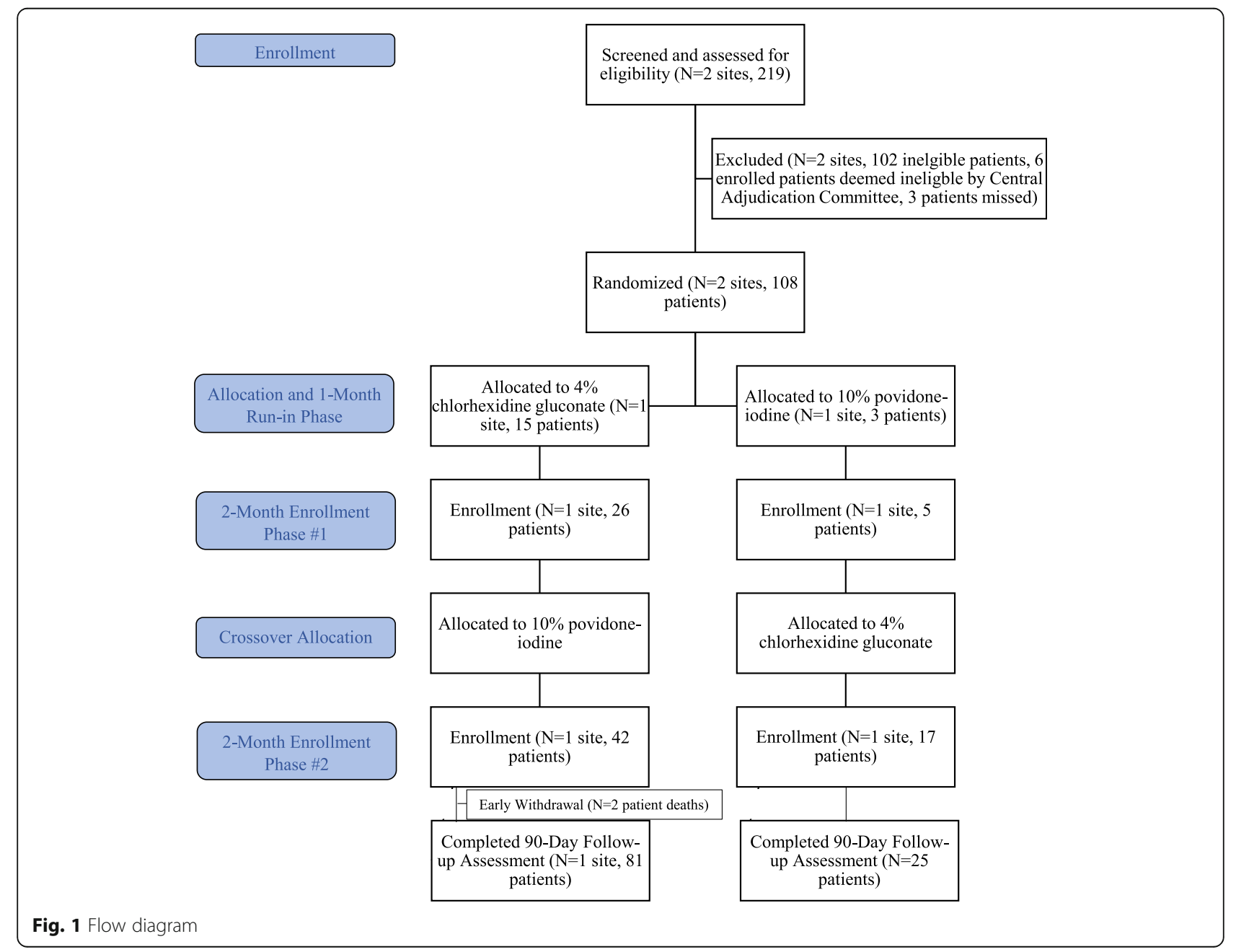


(91\%) were enrolled. Over the course of the two 2month enrollment phases (March 12, 2018 to July 17, 2018), HGH screened 52 patients, 26 of which were ineligible and excluded. Twenty-six screened patients were eligible, of which 25 (96\%) were enrolled. The reasons for exclusion are listed in Table 1. Overall, 92\% (95\% CI 85.9 to $96.4 \%$ ) of eligible patients at both sites were enrolled during the pilot phase, for a total of 108 participants. A total of 3 patients were missed and 6 patients were deemed ineligible by the Central Adjudication Committee (Table 1). Less than $1 \%$ of eligible patients did not provide informed consent. Consequently, enrollment rates were well above the established threshold of $75 \%$ of open fracture patients.

The majority of participants were male $(n=81,75.0 \%)$ and White $(n=68,63.0 \%)$ with an average age of 42 years (SD 17 years). Ninety-eight of the patients sustained one eligible open fracture (90.7\%) while 10 sustained two or three eligible open fractures (9.3\%). Thirty-nine of all 120 fractures (32.5\%) were of the upper extremities, while $81(67.5 \%)$ were lower extremity fractures, the most common of which were tibial and tibial shaft fractures $(n=49(40.8 \%$ of all 120 fractures)). According to the Gustilo Classification of Fracture system, 22 fractures were type I (18.3\%), 36 were type II (30.0\%), 41 were type IIIA (34.2\%), 13 were type IIIB (10.8\%), and 8 were type IIIC (6.7\%). Further details on participant demographics and fracture and injury characteristics are provided in Table 2.
Feasibility criteria 3: Treatment received as per cluster randomization

During the two 2-month enrollment phases, contamination rates were closely monitored. Ninety-seven percent $(81 / 83)$ of participants at STC were prepped correctly during their initial surgery. At HGH, 100\% (25/25) of participants were prepped correctly during their initial surgery. The two contaminations that did occur at STC were due to surgeon error. Overall, 98\% (106/108) (95\% CI 93.5 to $99.8 \%$ ) of participants across both pilot sites received the correct preparation solution during their initial surgery. Collectively, the two sites met the 95\% feasibility threshold for correct treatment (Table 3).

\section{Feasibility criteria 4: Participant follow-up}

Participant follow-up visits were also completed as per protocol as part of the pilot study. Ninety-seven percent $(81 / 83)$ of participants at STC completed follow-up at 90-day post-fracture surgery. Two of the 83 enrolled participants at STC died prior to completing follow-up. One hundred percent $(25 / 25)$ of participants at $\mathrm{HGH}$ completed follow-up at 90-day post-fracture surgery. Overall, 98\% (106/108) (95\% CI 93.5 to $99.8 \%)$ of participants across both pilot sites completed follow-up at 90-day post-fracture surgery, which exceeded the 95\% threshold for acceptable follow-up.

Feasibility criteria 5: Documentation of the primary outcome The primary outcome (SSI) was documented 100\% (95\% CI 96.6 to $100.0 \%$ ) accurately across both pilot sites.

Table 1 Reasons for exclusion

\begin{tabular}{|c|c|c|}
\hline Reasons for exclusion* & $\begin{array}{l}\text { Number of patients excluded } \\
\text { during screening } \\
n=102\end{array}$ & $\begin{array}{l}\text { Number of patients excluded } \\
\text { following adjudication } \\
n=6\end{array}$ \\
\hline $\begin{array}{l}\text { Did not receive or will not receive definitive fracture treatment } \\
\text { with a surgical implant(s) (e.g., internal fixation, external fixation, } \\
\text { joint prosthesis), } n(\%)\end{array}$ & $33(32.4)$ & $2(33.3)$ \\
\hline $\begin{array}{l}\text { Open fracture wound management did not include a formal } \\
\text { surgical debridement within } 72 \mathrm{~h} \text { of their injury, } n(\%)\end{array}$ & $21(20.6)$ & $2(33.3)$ \\
\hline $\begin{array}{l}\text { Likely problems, in the judgment of the study personnel, with } \\
\text { maintaining follow-up, } n(\%)\end{array}$ & $16(15.7)$ & N/A \\
\hline Incarceration, n (\%) & $8(7.8)$ & N/A \\
\hline Did not provide informed consent, $n(\%)$ & $7(6.9)$ & N/A \\
\hline Unable to obtain informed consent due to language barriers, $n(\%)$ & $6(5.9)$ & N/A \\
\hline $\begin{array}{l}\text { Received previous surgical debridement or management of their } \\
\text { open fracture(s) at a non-participating hospital or clinic, } n(\%)\end{array}$ & $4(3.9)$ & N/A \\
\hline Expected injury survival of less than 90 days, $n(\%)$ & $4(3.9)$ & N/A \\
\hline $\begin{array}{l}\text { Open fracture(s) managed outside of the participating orthopedic } \\
\text { service (e.g., hand fracture managed by plastic surgeon), } n(\%)\end{array}$ & $1(1.0)$ & N/A \\
\hline Burns at the fracture site, $n(\%)$ & $1(1.0)$ & N/A \\
\hline Not enrolled within 3 weeks of their fracture, $n(\%)$ & $1(1.0)$ & $1(16.7)$ \\
\hline Patient enrolled during run-in phase & N/A & $1(16.7)$ \\
\hline
\end{tabular}

*Please note that some patients were excluded for more than one reason. We present one reason per patient based on order of reason for exclusion in table 
Table 2 Participant demographics and fracture characteristics

\begin{tabular}{|c|c|}
\hline Characteristic & $\begin{array}{l}\text { Total } \\
n=108\end{array}$ \\
\hline Age, mean (SD) years & $41.9(17.1)$ \\
\hline \multicolumn{2}{|l|}{ Gender, $n(\%)$} \\
\hline Female & $27(25.0)$ \\
\hline Male & $81(75.0)$ \\
\hline \multicolumn{2}{|l|}{ BMI, $\left(\mathrm{kg} / \mathrm{m}^{2}\right), n(\%)$} \\
\hline Underweight $<18.5$ & $4(3.7)$ \\
\hline Normal weight 18.5-24.9 & $37(34.3)$ \\
\hline Overweight 25-29.9 & $39(36.1)$ \\
\hline Obese 30-39.9 & $21(19.4)$ \\
\hline Morbidly obese $\geq 40$ & $7(6.5)$ \\
\hline \multicolumn{2}{|l|}{ Ethnicity, $n(\%)$} \\
\hline White & $68(63.0)$ \\
\hline Black & $24(22.2)$ \\
\hline Hispanic/Latino & $8(7.4)$ \\
\hline East Asian & $2(1.9)$ \\
\hline Middle Eastern & $5(4.6)$ \\
\hline Indigenous & $1(0.9)$ \\
\hline Employment prior to injury, $n$ (\%) & $69(63.9)$ \\
\hline \multicolumn{2}{|l|}{ Highest level of education completed, $n$ (\%) } \\
\hline 8th grade or less & $1(1.0)$ \\
\hline 9th to 12 grade, no diploma & $10(9.3)$ \\
\hline $\begin{array}{l}\text { General education diploma or high school } \\
\text { graduate }\end{array}$ & $39(36.1)$ \\
\hline Some college, no degree & $23(21.3)$ \\
\hline Associates degree (2-year degree) & $8(7.4)$ \\
\hline Bachelors/college degree & $12(11.1)$ \\
\hline Some graduate work, no degree & $1(0.9)$ \\
\hline Graduate degree & $7(6.5)$ \\
\hline Professional degree & $3(2.8)$ \\
\hline Prefer not to answer & $4(3.7)$ \\
\hline \multicolumn{2}{|l|}{ Smoking history, $n(\%)$} \\
\hline No, never smoked & $42(38.9)$ \\
\hline Yes, previously smoked & $22(20.4)$ \\
\hline Yes, smokes currently & $44(40.7)$ \\
\hline \multicolumn{2}{|l|}{ Number of open fractures, $n$ (\%) } \\
\hline 1 & $98(90.7)$ \\
\hline 2 & $8(7.4)$ \\
\hline 3 & $2(1.9)$ \\
\hline Location of fracture, $n$ (\%) & $\begin{array}{l}n=120 \text { fractures } \\
\text { among } 108 \text { patients }\end{array}$ \\
\hline Clavicle & $1(0.8)$ \\
\hline Femur/femoral shaft & $15(12.5)$ \\
\hline Fibula & $2(1.7)$ \\
\hline Foot/ankle & 14 (11.7) \\
\hline
\end{tabular}

Table 2 Participant demographics and fracture characteristics (Continued)

\begin{tabular}{ll}
\hline Characteristic & $\begin{array}{l}\text { Total } \\
\boldsymbol{n}=\mathbf{1 0 8}\end{array}$ \\
\hline Hand & $7(5.8)$ \\
Humerus/humeral shaft & $13(10.8)$ \\
Patella & $1(0.8)$ \\
Radius/radial shaft & $10(8.3)$ \\
Tibia/tibial shaft & $49(40.8)$ \\
Ulna/ulnar shaft & $8(6.7)$ \\
Gustilo classification of fracture, $n$ (\%) & $n=120$ fractures \\
& among 108 patients \\
I & $22(18.3)$ \\
II & $36(30.0)$ \\
IIIA & $41(34.2)$ \\
IIIB & $13(10.8)$ \\
IIIC & $8(6.7)$
\end{tabular}

Percentages represent proportion out of total number of fractures by treatment group. Some participants sustained multiple fractures $B M I$ body mass index, $S D$ standard deviation

During the pilot trial, the CEO Methods Center conducted remote and on-site monitoring to ensure the pilot sites reported all events. As an error rate of less than $5 \%$ in the documentation of the primary outcome was required, the last feasibility criterion was met.

\section{Discussion}

The need for a definitive trials comparing preoperative antiseptic skin solutions is based on the conflicting results from existing trials, as well as the lack of research that compares antiseptic solutions in a specifically orthopedic surgical setting [10]. These inconsistent results leave the optimal antiseptic solution in doubt; in addition, results may differ across surgical settings. The risk of SSI is substantially greater in certain fracture populations (open fractures, closed lower extremity fractures, and pelvic fractures) due to the soft tissue trauma, wound contamination in open fractures, the increased risk of local vascular disruption, and the required surgery to fix the broken bones. Furthermore, the emergent nature of fracture surgery means that patients are unable to undergo other prophylactic skin care, such as $\mathrm{CHG}$ bathing, which is rendered to elective cases to reduce SSI. Additionally, the timing of prophylactic antibiotics may also fall beyond the recommended windows due to delays in getting to hospital; therefore, local antisepsis may become even more critical. The PREP-IT program includes two cluster randomized crossover trials which will definitely determine the optimal surgical preparation solution for this population. The Aqueous-PREP trial has been designed to study the effectiveness of a $10 \%$ povidone-iodine aqueous based solution versus $4 \%$ CHG 
Table 3 Feasibility outcomes

\begin{tabular}{|c|c|c|c|}
\hline Feasibility outcomes & Feasibility criteria & $\begin{array}{l}\text { Pilot study results } \\
n(\%)\end{array}$ & Feasibility criteria met \\
\hline $\begin{array}{l}\text { Implementation of the two trial } \\
\text { interventions during the 1-month } \\
\text { run-in period, } n(\%)\end{array}$ & $\begin{array}{l}\text { At least } 15 \text { eligible open fracture patients } \\
\text { with }>90 \% \text { of eligible patients receiving the } \\
\text { allocated antiseptic solution or a minimum } \\
\text { of } 1 \text { month in duration }\end{array}$ & $\begin{array}{l}\text { Total: } 18 / 18(100 \%) \\
\text { STC: } 15 / 15(100 \%) \\
\text { HGH: } 3 / 3(100 \%)\end{array}$ & Yes \\
\hline $\begin{array}{l}\text { Enrollment of participants across } \\
\text { the two planned } 2 \text {-month } \\
\text { enrollment phases, } n(\%)\end{array}$ & $\begin{array}{l}\text { Enrollment of }>75 \% \text { of open fracture patients } \\
\text { over each of the two } 2 \text {-month enrollment } \\
\text { periods }\end{array}$ & $\begin{array}{l}\text { Total: 108/117 (92\%) } \\
\text { STC: } 83 / 91 \text { (91\%) } \\
\text { HGH: } 25 / 26(96 \%)\end{array}$ & Yes \\
\hline $\begin{array}{l}\text { Participants receiving trial } \\
\text { interventions as per the cluster } \\
\text { randomization crossover } \\
\text { scheme, } n(\%)\end{array}$ & $\begin{array}{l}>95 \% \text { of participants receiving the treatment } \\
\text { as per the cluster randomization on their } \\
\text { initial surgery }\end{array}$ & $\begin{array}{l}\text { Total: } 106 / 108(98 \%) \\
\text { STC: } 81 / 83(97 \%) \\
\text { HGH: } 25 / 25(100 \%)\end{array}$ & Yes \\
\hline $\begin{array}{l}\text { Rates of Participant Follow-up, } \\
\text { n (\%) }\end{array}$ & $\begin{array}{l}\text { Participant follow-up of }>95 \% \text { at } 90 \text {-day } \\
\text { post-fracture surgery. }\end{array}$ & $\begin{array}{l}\text { Total: } 106 / 108(98 \%) \\
\text { STC: } 81 / 83(97 \%) \\
\text { HGH: } 25 / 25(100 \%)\end{array}$ & Yes \\
\hline $\begin{array}{l}\text { Accurate documentation of the } \\
\text { primary outcome, } n(\%)\end{array}$ & $\begin{array}{l}\text { Accurate documentation of }>95 \% \text { of the } \\
\text { primary outcome (SSI) (i.e., less than } 5 \% \text { error } \\
\text { rate in the documentation of the primary } \\
\text { outcome). }\end{array}$ & Total: 100\% & Yes \\
\hline
\end{tabular}

aqueous-based solution in reducing SSI. The PREPARE (Pragmatic Randomized Trial Evaluating Pre-Operative Alcohol Skin Solutions in Fractured Extremities) trial follows the same design as the Aqueous-PREP trial; however, it compares DuraPrep ${ }^{\mathrm{Tm}}$ versus ChloraPrep ${ }^{\mathrm{Tm}}$, which are alcohol-based solutions, in the management of open and closed fractures [5]. Similar to the Aqueous-PREP trial, the primary outcome of the PREP ARE trial is SSI and the secondary outcome is unplanned fracture-related reoperations within 12 months [5]. Two sister trials are required as there is a lack of consensus on the use of alcohol-based solutions in open fracture patients.

The Aqueous-PREP pilot trial, implemented at two orthopedic trauma centers, was used to test the feasibility of using a cluster randomized crossover study scheme to fill this gap in the existing research. All of the criteria corresponding to these five feasibility outcomes were met during the pilot phase of the Aqueous-PREP trial. The run-in phase was successful in regard to the number of eligible open-fracture patients received at each orthopedic study center. Although HGH treated only 3 eligible patients, this was anticipated as they are a smaller site in comparison to STC and their run-in period occurred in March when trauma volume is typically very low. During the 4-month enrollment period, $92 \%$ of patients across both sites were enrolled. As expected, enrollment at both clinical sites was higher during the second 2-month enrollment period due to seasonal variations in volume of traumatic injuries at both clinical sites. In response to the Central Adjudication Committee's determination that a number of participants enrolled at STC were ineligible, the team was retrained to ensure that additional enrollment errors would not occur in future. Informed consent was obtained from more than $99 \%$ of eligible patients. This high rate of consent is most likely attributable to the fact that the trial design allows for informed consent to take place after the intervention.

Compliance remained high during the run-in phase as well as over the course of the two enrollment periods. No contamination (i.e., the incorrect solution being applied as per cluster randomization) occurred during the run-in phase, and $99 \%$ of patients enrolled thereafter received the correct treatment during their initial surgery. Noncompliance rates increased to 5\% when accounting for the surgeries of patients who underwent repeat planned surgeries; however, the feasibility threshold of 95\% compliance did not apply to these additional procedures. The contaminations that did occur during the intervention phases were due to (1) surgeon error, (2) plastic surgery prepped the participant, and (3) surgery took place at another hospital. Half of the instances of noncompliance occurred within the first 2-month enrollment period and prior to the crossover between treatments. This suggests that the clinical sites' switch from the use of one antiseptic solution to another did not significantly increase contamination rates, and thus the crossover scheme was largely unproblematic.

Lastly, acceptable rates of patient follow-up and proper documentation of SSI, the primary outcome, were achieved across study sites. Ninety-eight percent of participants completed follow-up at 90-day post-fracture surgery, and the primary outcome was accurately documented for all participants. As such, all the success criteria were met, clearly suggesting that a definitive cluster randomization crossover study comparing preoperative antiseptic skin solutions is feasible. 
While the effectiveness of the case report forms (CRFs) used during this pilot study was not an official outcome, this feasibility study allowed for its informal assessment. Following the completion of the pilot phase and prior to initiation of a definitive trial, the AqueousPREP CRFs were significantly modified to facilitate straightforward and efficient data collection. The necessity of these changes demonstrates the importance of conducting pilot phases with limited enrollment before initiating large-scale definitive studies, as researchers may encounter protocol issues or areas of improvement not previously anticipated.

\section{Conclusions}

This pilot study, conducted at two clinical sites in two countries, confirmed the feasibility of a definitive study comparing antiseptic solutions in open fractures using a cluster randomized crossover trial design. This pilot represents a critical first step in the conduct of the Aqueous-PREP trial, especially in light of the fact that this trial follows a cluster randomized crossover design, which is novel in the field of orthopedic trauma surgery. Building upon the infrastructure, developed, and momentum and insights gained through the pilot study, we were able to successfully transition directly into the definitive trial phase of the Aqueous-PREP trial and the PREPARE trial.

\section{Abbreviations}

CEO: Center for Evidence-Based Orthopedics; CHG: Chlorhexidine gluconate; HGH: Hamilton Health Sciences General Site; SD: Standard deviation; SSI: Surgical site infection; STC: R Adams Cowley Shock Trauma Center

\footnotetext{
Acknowledgements

THE PREP-IT Investigators

Executive Committee: Gerard P. Slobogean (Principal Investigator, University of Maryland School of Medicine, Baltimore, MD); Sheila Sprague (Principal Investigator, McMaster University, Hamilton, ON); Jeffrey Wells (Patient Representative, Trauma Survivors Network, Falls Church, VA); Mohit Bhandar (Principal Investigator, McMaster University, Hamilton, ON) Steering Committee: Gerard P. Slobogean (Co-Chair, University of Maryland School of Medicine, Baltimore, MD); Mohit Bhandari (Co-Chair, McMaster University, Hamilton, ON); Sheila Sprague (Principal Investigator, McMaster University, Hamilton, ON); Jean-Claude D'Alleyrand (Walter Reed National Military Medical Center, Bethesda, MD); Anthony D. Harris (University of Maryland School of Medicine, Baltimore, MD); Daniel C. Mullins (University of Maryland, Baltimore, MD); Lehana Thabane (McMaster University, Hamilton, ON); Jeffrey Wells (Trauma Survivors Network, Falls Church, VA); Amber Wood (Association of periOperative Registered Nurses, Denver, CO)

Adjudication Committee: Gregory J. Della Rocca (Chair, University of Missouri, Columbia, MO): Anthony D. Harris, (University of Maryland School of Medicine, Baltimore, MD); Joan Hebden (University of Maryland, Baltimore, MD); Kyle J. Jeray (Greenville Health System, Greenville, SC); Lucas Marchand (University of Maryland, Baltimore, MD); Lyndsay M. O'Hara (University of Maryland School of Medicine, Baltimore, MD); Robert Zura (LSU Health, New Orleans, LA)

Data and Safety Monitoring Committee: Michael J. Gardner (Chair, Stanford University School of Medicine, Palo Alto, CA): Jenna Blasman (Patient Representative, Kitchener, ON); Jonah Davies (University of Washington, Seattle, WA); Stephen Liang (Washington University, St. Louis, MO); Monica Taljaard (Ottawa Hospital Research Institute, Ottawa, ON) Research Methodology Core: PJ Devereaux (McMaster University, Hamilton, ON); Gordon H. Guyatt (McMaster University, Hamilton, ON); Lehana Thabane
}

(McMaster University, Hamilton, ON); Diane Heels-Ansdell (McMaster University, Hamilton, ON)

Patient-Centered Outcomes Core: Debra Marvel (Patient Representative, Baltimore, MD); Jana Palmer (Patient Representative, Baltimore, MD); Jeffrey Wells (Patient, Trauma Survivors Network, Falls Church, VA); Jeff Friedrich (Editor, Slate Magazine, Washington DC); Daniel C. Mullins (University of Maryland, Baltimore, MD); Nathan N. O'Hara (University of Maryland School of Medicine, Baltimore, MD); Ms. Frances Grissom (Trauma Survivor Network, Baltimore, MD)

Orthopedic Surgery Core: Gregory J. Della Rocca (University of Missouri, Columbia, MO); I. Leah Gitajn (Dartmouth University, Hanover, NH); Kyle J. Jeray (Greenville Health System, Greenville, SC); Saam Morshed (San Francisco General Hospital, San Francisco, CA); Robert V. OToole (University of Maryland School of Medicine, Baltimore, MD); Bradley A. Petrisor (Hamilton Health Science, Hamilton, ON)

Operating Room Core: Megan Camara (R Adams Cowley Shock Trauma Center, Baltimore, MD); Franca Mossuto (Hamilton Health Science, Hamilton, $\mathrm{ON}$

Infectious Disease Core: Anthony D. Harris (University of Maryland School of Medicine, Baltimore, MD); Manjari G. Joshi (University of Maryland School of Medicine, Baltimore, MD)

Military Core: Jean-Claude D'Alleyrand (Walter Reed National Military Medical Center, Bethesda, MD); Justin Fowler (United States Army, USA); Jessica Rivera (San Antonio Military Medical Center, San Antonio, TX); Max Talbot (Canadian Armed Forces, Montreal, QC)

McMaster University Methods Center (Hamilton, ON): Sheila Sprague (Principal Investigator); Mohit Bhandari (Principal Investigator); Shannon Dodds (Research Coordinator); Alisha Garibaldi (Research Coordinator); Silvia Li (Research Coordinator); Uyen Nguyen (Research Coordinator); David Pogorzelski (Research Coordinator); Alejandra Rojas (Research Coordinator); Taryn Scott (Research Coordinator); Gina Del Fabbro (Research Assistant):

Olivia Paige Szasz (Research Assistant); Diane Heels-Ansdell (Statistician); Paula McKay (Manager)

University of Maryland School of Medicine Administrative Center (Baltimore, MD): Gerard P. Slobogean (Principal Investigator); Nathan N. O'Hara (Manager); Andrea Howe (Project Manager); Joshua Rudnicki (Project Manager); Haley Demyanovich (Project Manager); Kelly Little (Financial Manager)

University of Maryland School of Pharmacy, The PATIENTS Program

(Baltimore, MD): C. Daniel Mullins (Executive Director); Michelle Medeiros (Director of Research); Eric Kettering (Senior Instructional Technology and Dissemination Specialist); Diamond Hale (Project Manager)

PREP-IT Clinical Sites Lead Clinical Site (Aqueous-PREP and PREPARE)

University of Maryland School of Medicine, R Adams Cowley Shock Trauma Center, Baltimore, MD: Robert V. OToole, Jean-Claude D'Alleyrand, Andrew Eglseder, Aaron Johnson, Christopher Langhammer, Christopher Lebrun, Theodore Manson, Jason Nascone, Ebrahim Paryavi, Raymond Pensy, Andrew Pollak, Marcus Sciadini, Gerard P. Slobogean, Yasmin Degani, Haley K. Demyanovich, Andrea Howe, Nathan N. O'Hara, Katherine Joseph, Joshua Rudnicki, Megan Camara Aqueous-PREP and PREPARE Hamilton Health Sciences-General Site, Hamilton, ON: Brad A. Petrisor, Herman Johal, Bill Ristevski, Dale Williams, Matthew Denkers, Krishan Rajaratnam, Jamal Al-Asiri, Jordan Leonard, Francesc A. Marcano-Fernández*, Jodi Gallant, Federico Persico, Marko Gjorgjievski, Annie George IU Health Methodist Hospital, Indianapolis, IN: Roman M. Natoli, Greg E. Gaski, Todd O. McKinley, Walter W. Virkus, Anthony T. Sorkin, Jan P. Szatkowski, Joseph R. Baele, Brian H. Mullis, Lauren C. Hill, Andrea Hudgins, Methodist OR Core II Staff

San Antonio Military Medical Center, San Antonio, TX: Patrick Osborn, Justin Fowler, Sarah Pierrie, Eric Martinez, Joseph Kimmel

Prisma Health—Upstate, Greenville, SC: Kyle J. Jeray, John D. Adams, Michael L. Beckish, Christopher C. Bray, Timothy R. Brown, Andrew W. Cross, Timothy Dew, Gregory K. Faucher, Richard W. Gurich Jr, David E. Lazarus, S. John Millon, M. Jason Palmer, Scott E. Porter, Thomas M. Schaller, Michael S. Sridhar, John L. Sanders, L. Edwin Rudisill, Jr, Michael J. Garitty, Andrew S. Poole, Michael L. Sims, Clark M. Walker, Robert M. Carlisle II, Erin Adams Hofer, Brandon S. Huggins, Michael D. Hunter, William A. Marshall, Shea Bielby Ray, Cory D. Smith, Kyle M. Altman, Julia C. Bedard, Markus F. Loeffler, Erin R. Pichiotino, Austin A. Cole, Ethan J Maltz, Wesley Parker, T. Bennett Ramsey, Alex Burnikel, Michael Colello, Russell Stewart, Jeremy Wise, M. 
Christian Moody, Stephanie L. Tanner, Rebecca G. Snider, Christine E. Townsend, Kayla H. Pham, Abigail Martin, Emily Robertson University of California, San Francisco, San Francisco, CA: Saam Morshed, Theodore Miclau, Utku Kandemir, Meir Marmor, Amir Matityahu, R. Trigg McClellan, Eric Meinberg, David Shearer, Paul Toogood, Anthony Ding, Erin Donohue, Tigist Belaye, Eleni Berhaneselase, Alexandra Paul*, Kartik Garg Aqueous-PREP

McGovern Medical School at UTHealth Houston, Houston, TX: Joshua L. Gary, Stephen J Warner, John W. Munz, Andrew M. Choo, Timothy S. Achor, Milton L. "Chip" Routt, Mayank Rao, Guillermo Pechero, Adam Miller* University of Florida, Gainesville, FL: Jennifer E. Hagen, Matthew Patrick, Richard Vlasak, Thomas Krupko, Kalia Sadasivan*, Chris Koenig, Daniel Bailey*, Daniel Wentworth*, Chi Van, Justin Schwartz

The CORE Institute, Phoenix, AZ: Niloofar Dehghan, Clifford B Jones*, J Tracy Watson, Michael McKee, Ammar Karim*, Michael Talerico, Debra L Sietsema, Alyse Williams, Tayler Dykes

Vanderbilt Medical Center, Nashville, TN: William T Obremskey, Amir Alex Jahangir, Manish Sethi, Robert Boyce, Daniel J. Stinner, Phillip Mitchell, Karen Trochez, Andres Rodriguez*, Vamshi Gajari, Elsa Rodriguez, Charles Pritchett Banner University Medical Center-Tucson, Tucson, AZ: Christina Boulton, Jason Lowe, Jason Wild*, John T. Ruth, Michel Taylor, Andrea Seach, Sabina Saeed, Hunter Culbert, Alejandro Cruz, Thomas Knapp*, Colin Hurkett*, Maya Lowney

Wright State University, Dayton, OH: Michael Prayson, Indresh

Venkatarayappa, Brandon Horne, Jennifer Jerele, Linda Clark

Hospital Universitari Parc Tauli, Barcelona, Spain: Francesc Marcano-

Fernández, Montsant Jornet-Gibert, Laia Martínez-Carreres, David Martí-Garín, Jorge Serrano-Sanz,

Joel Sánchez-Fernández, Matsuyama Sanz-Molero, Alejandro Carballo, Xavier Pelfort, Francesc Acerboni-Flores, Anna Alavedra-Massana, Neus AngladaTorres, Alexandre Berenguer, Jaume Cámara-Cabrera, Ariadna CaparrosGarcía, Ferran Fillat-Gomà, Ruben Fuentes-López, Ramona Garcia-Rodriguez, Nuria Gimeno-Calavia, Guillem Graells-Alonso, Marta Martínez-Álvarez, Patricia Martínez-Grau, Raúl Pellejero-García, Ona Ràfols-Perramon, Juan Manuel Peñalver, Mònica Salomó Domènech, Albert Soler-Cano, Aldo Velasco-Barrera, Christian Yela-Verdú, Mercedes Bueno-Ruiz, Estrella Sánchez-Palomino Vall d'Hebron Hospital, Barcelona, Spain: Ernesto Guerra, Yaiza García PREPARE

MetroHealth Medical Center, Cleveland, OH: Nicholas M. Romeo, Heather A Vallier, Mary A Breslin*, Joanne Fraifogl, Eleanor S Wilson*, Leanne K Wadenpfuhl*, Paul G. Halliday

Fraser Health Authority/Royal Columbian Hospital, New Westminster, BC: Darius G. Viskontas, Kelly L. Apostle, Dory S. Boyer, Farhad O. Moola, Bertrand H. Perey, Trevor B. Stone, H. Michael Lemke, Mauri Zomar, Ella Spicer, Chen "Brenda" Fan, Kyrsten Payne

Carolinas Medical Center, Atrium Health Musculoskeletal Institute, Charlotte, NC: Kevin Phelps, Michael Bosse, Madhav Karunakar, Laurence Kempton, Stephen Sims, Joseph Hsu, Rachel Seymour, Christine Churchill, Claire Bartel, Robert Miles Mayberry, Maggie Brownrigg, Cara Girardi, Ada Mayfield Inova Fairfax Medical Campus, Falls Church, VA: Robert A. Hymes, Cary C. Schwartzbach, Jeff E. Schulman, A. Stephen Malekzadeh, Michael A. Holzman, Lolita Ramsey, James S. Ahn, Farhanaz Panjshiri*, Sharmistha Das, Antoinisha D. English, Sharon M. Haaser, Jaslynn A. N. Cuff

Wake Forest Baptist Health, Winston-Salem, NC: Holly Pilson, Eben A. Carroll, Jason J. Halvorson, Sharon Babcock, J. Brett Goodman, Martha B. Holden, Debra Bullard, Wendy Williams

University of Utah, Salt Lake City, Utah: Thomas F. Higgins, Justin M. Haller, David L. Rothberg, Ashley Neese, Mark Russell

Dartmouth-Hitchcock Medical Center, Lebanon, NH: I. Leah Gitajn, Marcus Coe, Kevin Dwyer, Devin S. Mullin, Clifford A. Reilly, Peter DePalo, Amy E. Hall Massachusetts General Hospital, Boston, MA: Marilyn Heng, Mitchel B. Harris, R. Malcolm Smith, David W. Lhowe, John G. Esposito, Mira Bansal University of Mississippi Medical Center, Jackson, MS: Patrick F. Bergin, George V. Russell, Matthew L. Graves, John Morellato, Heather K. Champion, Leslie N. Johnson, Sheketha L. McGee, Eldrin L. Bhanat University of Pennsylvania, Philadelphia, PA: Samir Mehta, Derek Donegan, Jaimo Ahn, Annamarie Horan, Mary Dooley, Ashley Kuczinski, Ashley Iwu Sanford Health, Sioux Falls, SD: David Potter, Robert VanDemark III, Branden Pfaff, Troy Hollinsworth Brigham Women's Hospital, Boston, MA: Michael J. Weaver, Arvind G. von Keudell, Michael F. McTague, Elizabeth M. Allen
University of Maryland Prince George's Capital Region Health: Cheverly MD: Todd Jaeblon, Robert Beer, Haley K. Demyanovich

Duke University Hospital, Durham, NC: Mark J. Gage, Rachel M. Reilly, Cindy Sparrow

*Individual is no longer actively working on the Aqueous-PREP and/or PREPARE trial

\section{Authors' contributions}

All authors listed under the Acknowledgments section reviewed the manuscript and provided critical input regarding its intellectual content. The authors read and approved the final manuscript.

\section{Funding}

The Aqueous-PREP trial is funded by the US Department of Defense (W81XWH-17-1-070) and the CIHR (Foundation Grant). McMaster University Surgical Associates funded start-up activities at the Methods Center and The Physician Services Incorporated provided funding to the Methods Center and Hamilton Health Sciences for the Aqueous-PREP trial. Study funders have no role in the study design, collection, management, analysis, and interpretation of data.

\section{Availability of data and materials}

All data generated in this study will be analyzed upon completion of the participant follow-up during the definitive phase of the trial and will be published in a journal article in future.

\section{Ethics approval and consent to participate}

This protocol, the consent form template, and the case report forms have been reviewed and approved by the Hamilton Integrated Research Ethics Board and the Advarra Institutional Review Board (formerly Chesapeake Institutional Review Board). Prior to commencement of the study the clinical site must provide the Methods Center with a copy of the ethics board approval.

\section{Consent for publication}

Not applicable.

\section{Competing interests}

The authors declare no conflict of interest with regards to this study.

\section{Author details}

'Division of Orthopedic Surgery, Department of Surgery, McMaster University, Hamilton, Ontario, Canada. ${ }^{2}$ Department of Health Research Methods, Evidence, and Impact, McMaster University, Hamilton, Ontario, Canada. ${ }^{3} \mathrm{R}$ Adams Cowley Shock Trauma Center, Department of Orthopedics, University of Maryland School of Medicine, Baltimore, MD, USA.

\section{Received: 21 September 2020 Accepted: 16 February 2021}

\section{Published online: 01 March 2021}

\section{References}

1. Darouiche RO, Wall MJ, Itani KMF, et al. Chlorhexidine-alcohol versus povidone-iodine for surgical-site antisepsis. N Engl J Med. 2010;362(1):18-26.

2. Tuuli MG, Liu J, Stout MJ, et al. A randomized trial comparing skin antiseptic agents at cesarean delivery. N Engl J Med. 2016;374(7):647-55.

3. Swenson BR, Sawyer RG. Importance of alcohol in skin preparation protocols. Infect Control Hosp Epidemiol. 2010;31(9):977.

4. Swenson BR, Hedrick TL, Metzger R, Bonatti H, Pruett TL, Sawyer RG. Effects of preoperative skin preparation on postoperative wound infection rates: a prospective study of 3 skin preparation protocols. Infect Control Hosp Epidemiol. 2009;30(10):964-71.

5. Program of Randomized Trials to Evaluate Pre-operative Antiseptic Skin Solutions in Orthopaedic Trauma (PREP-IT) Investigators, Slobogean GP, Sprague S, et al. Effectiveness of iodophor vs chlorhexidine solutions for surgical site infections and unplanned reoperations for patients who underwent fracture repair: The PREP-IT master protocol. JAMA Netw Open. 2020;3(4):e202215.

6. Eldridge $\mathrm{SM}$, Chan CL, Campbell MJ, et al. CONSORT 2010 statement: extension to randomised pilot and feasibility trials. BMJ. 2016;355:15239.

7. McDonnell G, Russell AD. Antiseptics and disinfectants: activity, action, and resistance. Clin Microbiol Rev. 1999;12(1):147-79. 
8. Campbell MK, Piaggio G, Elbourne DR, Altman DG, CONSORT Group. Consort 2010 statement: extension to cluster randomised trials. BMJ. 2012; 345:e5661.

9. Arnup SJ, Forbes AB, Kahan BC, Morgan KE, McKenzie JE. Appropriate statistical methods were infrequently used in cluster-randomized crossover trials. J Clin Epidemiol. 2016;74:40-50.

10. Wade RG, Burr NE, McCauley G, Bourke G, Efthimiou O. The comparative efficacy of chlorhexidine gluconate and povidone-iodine antiseptics for the prevention of infection in clean surgery: a systematic review and network meta-analysis. Ann Surg. 2020. Online ahead of print.

\section{Publisher's Note}

Springer Nature remains neutral with regard to jurisdictional claims in published maps and institutional affiliations.

Ready to submit your research? Choose BMC and benefit from:

- fast, convenient online submission

- thorough peer review by experienced researchers in your field

- rapid publication on acceptance

- support for research data, including large and complex data types

- gold Open Access which fosters wider collaboration and increased citations

- maximum visibility for your research: over $100 \mathrm{M}$ website views per year

At BMC, research is always in progress.

Learn more biomedcentral.com/submissions 